\title{
Risk of Bleeding Associated with Antidepressant Drugs: The Competitive Impact of Antithrombotics in Quantitative Signal Detection
}

\author{
René Zeiss $^{1}$ (D) . Christoph Hiemke ${ }^{2} \cdot$ Carlos Schönfeldt-Lecuona $^{1} \cdot$ Bernhard J. Connemann ${ }^{1} \cdot$ Maximilian Gahr $^{1}$
}

Accepted: 11 May 2021 / Published online: 11 June 2021

(c) The Author(s) 2021

\begin{abstract}
Background To date, disproportionality analysis has been unable to demonstrate the increased bleeding risk associated with antidepressant drugs, especially selective serotonin reuptake inhibitors.

Objective We hypothesised that a potential signal for an increased bleeding risk may be mitigated by the effects of agents other than antidepressant drugs that are strongly associated with haemorrhages, especially antithrombotics. In addition, we investigated if the use of more specific search terms of the Medical Dictionary for Regulatory Activities facilitates the detection of signals.

Methods Pharmacovigilance data from the Uppsala Monitoring Centre were used to calculate substance-specific reporting odds ratios (RORs) for all types of bleeding and gastrointestinal bleeding. Reporting odds ratios were calculated with and without antithrombotic comedication.

Results Regarding any type of bleeding, no signals were found in association with antidepressant drugs. Concerning upper gastrointestinal bleeding, signals were found related to citalopram (ROR: 1.56 [95\% confidence interval 1.11-2.20]) and escitalopram (ROR: 1.52 [95\% confidence interval 1.03-2.25]). After removal of reports related to antithrombotics, these signals could no longer be detected, but a new signal related to St. John's Wort associated with haemorrhages was found (ROR: 1.50 [95\% confidence interval 1.21-1.86]).

Conclusions Antithrombotics seem unlikely to have a major impact on the detection of the bleeding risk of antidepressant drugs. The different categorisation of adverse drug reactions regarding the strength of a causal relationship between a drug and an event in the database may be relevant for this negative finding.
\end{abstract}

\section{Introduction}

Post-marketing surveillance is of eminent importance for drug safety [1]. Numerous adverse drug reactions (ADRs), especially rare ADRs, cannot be identified in clinical trials and premarket approvals because of a limited number of trial participants or the exclusion of older or severely ill participants. Individual case safety reports (ICSRs) and pharmacovigilance databases play a major role in generating 'signals', which may serve as a starting point for further

René Zeiss

rene.zeiss@uni-ulm.de

1 Department of Psychiatry and Psychotherapy III, University of Ulm, Leimgrubenweg 12-14, 89075 Ulm, Germany

2 Department of Psychiatry and Psychotherapy, University Medical Center of Mainz, Mainz, Germany

\section{Key Points}

We found evidence of an increased bleeding risk for upper gastrointestinal bleeding related to several selective serotonin reuptake inhibitors.

In this study, antithrombotics did not have a major impact on signal detection regarding the bleeding risk related to antidepressant drugs.

The competition bias should be considered with caution, if the competing substances may have an impact in terms of a drug-drug interaction.

Other factors, such as the different categorisation of suspected reports of adverse drug reactions regarding the strength of a causal relationship between a drug and an event in the database, might also be relevant for signal detection. 
drug safety investigations [2]. The definition of a signal has developed and changed over the course of time. According to Meyboom et al., a signal consists of a hypothesis, together with data and arguments [3]. In disproportionality analysis, a signal is characterised by the reporting of events that are statistically disproportionate within the respective database [4]. Spontaneous reporting databases and disproportionality analyses are effective tools in the field of pharmacovigilance $[5,6]$. Apart from the already mentioned possibility to detect signals for rare ADRs, disproportionality analyses offer the possibility to investigate the likelihood of drug-drug interactions or to verify pharmacological hypotheses such as the association between different receptor occupancies and antipsychotic-induced movement disorders [7, 8]. Despite the key role of disproportionality analysis in drug safety, there are several limitations and biases that need to be considered. Some of the limitations are for example the phenomenon of under-reporting $[9,10]$, the notoriety bias [11] or the Weber effect [9]. Another major bias in disproportionality analysis is competition bias [12]: it can occur both in the form of a competition between drugs or between events/ADRs. Either way, the competition bias may mask potential signals and complicate signal detection $[13,14]$. The competition bias may occur, if an ADR is commonly reported related to a substance other than the substance of interest [12]. As a consequence, this impedes the detection of a signal related to the substance of interest. The competition bias may also occur, if a specific ADR related to a drug is very frequently reported, thus masking other less frequently reported ADRs related to the same drug, as in the case of statins and muscle-related ADRs. This type of competition bias may mask signals for well-known ADRs such as sexual dysfunction, cholestasis and jaundice, and skin reactions [14].

In psychopharmacology, antidepressant drugs (ADs) and in particular ADs with influence on the serotonergic system, especially selective serotonin reuptake inhibitors (SSRIs), are first-line drugs for the pharmacological treatment of depressive disorders [15]. Regarding SSRIs, there is evidence of a clinically relevant increased risk for bleeding [16]. Pharmacodynamic and pharmacokinetic interactions might contribute to the increased bleeding risk, the cytochromes P450 may affect and may be affected by both substance classes, ADs (especially fluoxetine and fluvoxamine) and antithrombotics such as warfarin or direct-acting oral anticoagulants [17]. Furthermore, one major mechanism for the increased bleeding risk of ADs is presumably serotonin uptake inhibition into platelets by inhibition of the serotonin transporters and the resulting functional impairment in blood coagulation [18, 19]. As recent findings suggest a quantitative relationship between a drug's binding affinity for the serotonin transporter and the magnitude of the increase in the bleeding risk [20,21], all substances that inhibit (re)uptake of serotonin may theoretically be associated with an increased bleeding risk. In addition, there are findings suggesting a localisation-specific increased risk of bleeding in association with SSRIs in the context of breast biopsies [22], perioperative bleeding [23, 24] and intracranial haemorrhage [21]. There is also major evidence suggesting that the risk of bleeding related to SSRIs seems to be particularly increased in the upper gastrointestinal tract [25, 26]. However, the risk of bleeding related to SSRIs seems to be relatively small [27] and particularly relevant in combination with certain substances, such as antithrombotics [28]. Yet, many observational studies suggest a considerably increased risk for bleeding related to SSRIs [16]. However, until now this could not be verified with methods of pharmacovigilance, particularly by disproportionality analysis [29, 30]. The reason might be the aforementioned competition bias, as bleeding is an ADR commonly associated with antithrombotics and the insufficient consideration of specific anatomical localisations of bleedings. Therefore, in the current study, we investigated, if (1) the competition bias (considering antithrombotics) may be relevant for detecting the bleeding risk related to ADs with disproportionality analysis, and if (2) focusing on specific anatomical localisations that are known to be associated with a particular risk for AD-related bleeding (e.g. upper gastrointestinal tract) may facilitate the detection of a risk of bleeding related to ADs by using disproportionality analysis.

\section{Methods}

\subsection{Database}

We analysed data from VigiBase ${ }^{\mathrm{TM}}$, the World Health Organization global database of ICSRs maintained by the Uppsala Monitoring Centre (UMC). At the time of the study, the database contained more than 19 million reports of suspected adverse effects. The member countries share the reports gathered in the national pharmacovigilance centres by sending them to the UMC. Each submitted report is evaluated according to predefined quality criteria. The data obtained in the national pharmacovigilance centres are heterogenous, as their origin may vary depending on the respective source, for example health professionals, patients as well as pharmaceutical companies. Likewise, the causality assessment systems may differ depending on the reporting national pharmacovigilance centre. Finally, it is important to consider that the ADR data and their implications do not represent the opinion of the UMC or the World Health Organization. 


\subsection{Database Query}

The study period was defined from 1968 to the index date of the database query (September 2018). For the database search, terms from the Standardised Medical Dictionary for Regulatory Activities (MedDRA) query (SMQ) Version 21.1 were used. MedDRA is a standardised international medical terminology, which is, inter alia, designed for safety monitoring of medicinal products [31]. MedDRA is organised in a hierarchical structure of different terms. Standardised MedDRA Queries are validated sets of MedDRA terms used to ensure comparability of drug safety investigations in pharmacovigilance.

\subsection{Applied Search Terms and Evaluated Substances}

The applied SMQs were "Haemorrhages" (narrow scope) and "Gastrointestinal haemorrhage" and the preferred term (PT) "Upper gastrointestinal haemorrhage". All reports entered in Vigibase ${ }^{\mathrm{TM}}$ at the time of the database query were considered. We retrieved the numbers of ICSRs related to defined drug/event combinations. Data were retrieved for the following antidepressant substances related to the category suspected/interacting: agomelatine, amitriptyline, bupropion, citalopram, clomipramine, doxepin, duloxetine, escitalopram, fluoxetine, fluvoxamine, hypericum perforatum, imipramine, maprotiline, milnacipran, mirtazapine, moclobemide, nortriptyline, paroxetine, reboxetine, sertraline, tianeptine, tranylcypromine, trazodone, trimipramine and venlafaxine. The category "suspected/interacting" was chosen to increase the specificity of the reports and the probability of a causal relationship between the occurrence of the respective event and the respective substance. The categorisation of a substance concerning the likelihood of a causal relationship with the respective event ("suspected/interacting" or "concomitant") is made by the reporter of the ADR. Signal detection was first carried out in the complete database; in a second step, the competition bias was considered, removing all reports related to competitive substances, namely "antithrombotic agents" as listed in the Anatomical Therapeutic Chemical Classification System (ATC) B01A, from the database. The antithrombotic agents in ATC B01A were selected as competitors because they are a group of drugs showing haemorrhages as a type A reaction [14, 32]. Type A reactions are augmented pharmacological effects of the respective drug and may therefore occur very frequently as ADRs.

\subsection{Statistical Evaluation}

The calculation of the reporting odds ratio (ROR) as a tool for disproportionality analysis is a frequently used approach for signal generation [9, 33, 34]. The ROR is calculated based on a case/non-case approach, whereby the reporting odds of one particular event related to a given drug are compared with the reporting odds of all other events related to the respective drug. A signal is defined when the lower limit of the $95 \%$ confidence interval (CI) of the ROR is greater than $1.0[35,36]$. Reporting odds ratios were only calculated when the number of ICSRs for a drug/ADR pair exceeded the minimal threshold of 3 .

In the present study, three case/non-case analyses were performed. Cases were defined as ICSRs associated with the SMQ "Haemorrhages" in the first analysis; in the second analysis, cases were defined as ICSRs associated with the SMQ "Gastrointestinal haemorrhage", and in the third analysis, cases were defined as ICSRs associated with the PT "Upper gastrointestinal haemorrhage"; all other ICSRs were considered as non-cases. All analyses were performed with Microsoft ${ }^{\circledR}$ Excel Version 16.16.8 (One Microsoft Way, Redmond, WA, USA).

\section{Results}

At the index date (September 2018), there were 18,409,119 ICSRs in VigiBase of which 975,879 were associated with the SMQ "Haemorrhages" (narrow scope), 254,366 with SMQ "Gastrointestinal haemorrhage" and 10,798 with the PT "Upper gastrointestinal haemorrhage". After removing all reports associated with an antithrombotic (according to ATC code B01A), there was a total amount of 16,917,158 reports of which 635,987 were associated with the SMQ "Haemorrhages" (narrow scope), 138,817 for SMQ "Gastrointestinal haemorrhage" and 3509 for the PT "Upper gastrointestinal haemorrhage". Table 1 shows the RORs for SMQ "Haemorrhages" for the whole dataset with and without consideration of a potential competition bias by antithrombotics. Table 2 shows the RORs with and without consideration of the competition bias for SMQ "Gastrointestinal haemorrhage", Table 3 shows the data for the PT "Upper gastrointestinal haemorrhage". None of the substances triggered a signal in the whole dataset related to any of the two SMQs. Regarding the PT "Upper gastrointestinal haemorrhage", there was a signal for citalopram (ROR: 1.56 [95\% CI 1.11-2.20]) and escitalopram (ROR: 1.52 [95\% CI 1.03-2.25]). These two signals disappeared after removing reports related to antithrombotics. Only for Hypericum perforatum (ROR: 1.50 [95\% CI 1.21-1.86]), a new signal associated with the SMQ "Haemorrhages" was found after removing antithrombotics from the database. 
Table 1 RORs related to antidepressant drugs and the SMQ "Haemorrhages" (narrow scope) with and without consideration of the hypothesised competition bias with antithrombotic comedication

\begin{tabular}{|c|c|c|}
\hline \multirow[t]{2}{*}{ Substance } & \multicolumn{2}{|l|}{ ROR $(95 \% \mathrm{CI})$} \\
\hline & $\begin{array}{l}\text { ROR without consideration of the } \\
\text { competition bias }\end{array}$ & $\begin{array}{l}\text { ROR with consideration } \\
\text { of the competition bias }\end{array}$ \\
\hline Agomelatine & $0.33(0.25-0.43)$ & $0.45(0.34-0.59)$ \\
\hline Amitriptyline & $0.35(0.32-0.38)$ & $0.43(0.39-0.48)$ \\
\hline Bupropion & $0.32(0.31-0.34)$ & $0.45(0.42-0.47)$ \\
\hline Citalopram & $0.85(0.81-0.89)$ & $0.84(0.79-0.90)$ \\
\hline Clomipramine & $0.41(0.35-0.46)$ & $0.55(0.47-0.63)$ \\
\hline Doxepin & $0.34(0.29-0.41)$ & $0.46(0.38-0.56)$ \\
\hline Duloxetine & $0.59(0.56-0.62)$ & $0.72(0.68-0.75)$ \\
\hline Escitalopram & $0.88(0.83-0.93)$ & $0.86(0.80-0.92)$ \\
\hline Fluoxetine & $0.74(0.71-0.76)$ & $0.96(0.92-1.00)$ \\
\hline Fluvoxamine & $0.49(0.43-0.55)$ & $0.65(0.57-0.73)$ \\
\hline Hypericum perforatum & $1.06(0.86-1.30)$ & $1.50(1.21-1.86)$ \\
\hline Imipramine & $0.31(0.26-0.38)$ & $0.44(0.36-0.54)$ \\
\hline Lithium & $0.22(0.20-0.25)$ & $0.32(0.28-0.36)$ \\
\hline Maprotiline & $0.32(0.25-0.41)$ & $0.45(0.35-0.57)$ \\
\hline Milnacipran & $0.43(0.35-0.52)$ & $0.54(0.43-0.67)$ \\
\hline Mirtazapine & $0.31(0.28-0.34)$ & $0.36(0.32-0.40)$ \\
\hline Moclobemide & $0.23(0.17-0.30)$ & $0.32(0.24-0.43)$ \\
\hline Nortriptyline & $0.35(0.29-0.42)$ & $0.47(0.39-0.56)$ \\
\hline Paroxetine & $0.63(0.60-0.66)$ & $0.71(0.68-0.74)$ \\
\hline Reboxetine & $0.32(0.24-0.44)$ & $0.46(0.34-0.63)$ \\
\hline Sertraline & $0.67(0.64-0.70)$ & $0.82(0.78-0.86)$ \\
\hline Tianeptine & $0.30(0.21-0.44)$ & $0.41(0.27-0.61)$ \\
\hline Tranylcypromine & $0.72(0.57-0.92)$ & $0.99(0.77-1.27)$ \\
\hline Trazodone & $0.28(0.25-0.32)$ & $0.38(0.33-0.43)$ \\
\hline Trimipramine & $0.41(0.31-0.55)$ & $0.54(0.40-0.74)$ \\
\hline Venlafaxine & $0.58(0.56-0.61)$ & $0.73(0.69-0.77)$ \\
\hline
\end{tabular}

$C I$ confidence interval, $R O R$ reporting odds ratio, SMQ Standardised Medical Dictionary for Regulatory Activities Queries

Signals are written in italic

\section{Discussion}

In the present article, we investigated if comedication with antithrombotics could be a bias for the detection of the bleeding risk related to ADs especially SSRIs in disproportionality analyses. Using the largest worldwide pharmacovigilance databases, we identified cases of bleeding events and removed all reports associated with antithrombotics. Furthermore, we investigated if an association with a localisation-specific bleeding risk is detectable by using different specific terms from the MedDRA. To the best of our knowledge, this is the first study investigating a possible influence of antithrombotics on signal detection regarding the bleeding risk associated with ADs.
Apart from Hypericum perforatum, no additional signals were detected by considering a possible competition related to antithrombotics. However, findings of previous studies suggested an influence of the competition bias on bleeding events for a hypothetic drug after removal of reports related to antiplatelet agents, anticoagulant agents and thrombolytic agents from the database [12]. There are several factors possibly explaining the absent influence of the competition bias in our study. First, the described bleeding risk for ADs is considered low and, in many cases, the respective bleeding symptoms are not severe $[19,27]$. Usually ADRs are less likely to be reported, if they do not feature a fatal outcome, are trivial, or if there is uncertainty about the drug causing the ADR [10]. A major point to consider is that 
Table 2 RORs related to antidepressant drugs and the SMQ "Gastrointestinal haemorrhage" with and without consideration of the competition bias with antithrombotic comedication

\begin{tabular}{|c|c|c|}
\hline \multirow[t]{2}{*}{ Substance } & \multicolumn{2}{|l|}{$\operatorname{ROR}(95 \% \mathrm{CI})$} \\
\hline & $\begin{array}{l}\text { ROR without consideration of the com- } \\
\text { petition bias }\end{array}$ & $\begin{array}{l}\text { ROR with consideration } \\
\text { of the competition bias }\end{array}$ \\
\hline Agomelatine & $0.24(0.14-0.44)$ & $0.40(0.22-0.74)$ \\
\hline Amitriptyline & $0.25(0.20-0.30)$ & $0.34(0.27-0.43)$ \\
\hline Bupropion & $0.24(0.21-0.27)$ & $0.37(0.32-0.43)$ \\
\hline Citalopram & $0.95(0.87-1.04)$ & $0.83(0.73-0.94)$ \\
\hline Clomipramine & $0.13(0.08-0.21)$ & $0.22(0.14-0.36)$ \\
\hline Doxepin & $0.18(0.11-0.29)$ & $0.26(0.15-0.45)$ \\
\hline Duloxetine & $0.62(0.56-0.67)$ & $0.86(0.78-0.95)$ \\
\hline Escitalopram & $0.84(0.75-0.94)$ & $0.86(0.75-1.00)$ \\
\hline Fluoxetine & $0.50(0.46-0.54)$ & $0.67(0.61-0.74)$ \\
\hline Fluvoxamine & $0.39(0.29-0.51)$ & $0.55(0.40-0.74)$ \\
\hline Hypericum perforatum & $0.56(0.32-0.96)$ & $0.69(0.36-1.32)$ \\
\hline Imipramine & $0.11(0.06-0.21)$ & $0.17(0.08-0.33)$ \\
\hline Lithium & $0.20(0.16-0.26)$ & $0.35(0.28-0.44)$ \\
\hline Maprotiline & $0.10(0.04-0.23)$ & $0.10(0.03-0.31)$ \\
\hline Milnacipran & $0.30(0.19-0.48)$ & $0.44(0.26-0.75)$ \\
\hline Mirtazapine & $0.21(0.16-0.26)$ & $0.25(0.19-0.34)$ \\
\hline Moclobemide & $0.16(0.08-0.31)$ & $0.26(0.13-0.51)$ \\
\hline Nortriptyline & $0.17(0.10-0.28)$ & $0.27(0.16-0.45)$ \\
\hline Paroxetine & $0.46(0.42-0.50)$ & $0.46(0.41-0.52)$ \\
\hline Reboxetine & $0.22(0.10-0.46)$ & $0.33(0.15-0.73)$ \\
\hline Sertraline & $0.54(0.49-0.59)$ & $0.68(0.61-0.75)$ \\
\hline Tianeptine & $0.13(0.04-0.39)$ & $0.24(0.08-0.74)$ \\
\hline Tranylcypromine & $0.20(0.09-0.49)$ & $0.36(0.15-0.86)$ \\
\hline Trazodone & $0.20(0.15-0.26)$ & $0.28(0.21-0.39)$ \\
\hline Trimipramine & $0.13(0.05-0.36)$ & $0.18(0.06-0.56)$ \\
\hline Venlafaxine & $0.49(0.44-0.54)$ & $0.66(0.59-0.74)$ \\
\hline
\end{tabular}

$C I$ confidence interval, $R O R$ reporting odds ratio, $S M Q$ Standardised Medical Dictionary for Regulatory Activities Queries bleeding events associated with ADs are frequently associated with comedication, inter alia antithrombotics [19]. An increased risk of non-gastrointestinal bleeding [37] and upper gastrointestinal bleeding [38] related to SSRI in combination with anticoagulative drugs was reported. Moreover, there is evidence of an increased risk of intracranial haemorrhage related to ADs with strong inhibition of serotonin reuptake (respectively high binding affinity to the serotonin transporter), particularly in combination with oral anticoagulants [21]. There might be a relevant pharmacodynamic interaction between ADs and antithrombotics involving, inter alia, the cytochrome p450 system resulting in an increased bleeding risk. Several case reports describe a potential interaction not only between ADs and warfarin, but also with direct oral anticoagulants [17, 39]. As we removed all reports associated with antithrombotics from the database, the number of reports of haemorrhages related to ADs was strongly reduced because many reports were associated with an antithrombotic comedication, thus affecting the results of our disproportionality analysis. This might especially affect the analysis of gastrointestinal bleeding events, as not only warfarin but also direct oral anticoagulants are strongly associated with an increased risk of gastrointestinal bleeding as recent analyses of drug safety databases demonstrated [40, 41]. Therefore, we hypothesise a major impact of the well-known aspect of a drug-drug interaction regarding the bleeding risk related to SSRIs (in terms of two substance groups are associated with an increased risk for the ADR of interest), which cannot be adequately considered by using the present methodology. Another reason for the missing influence of the competition bias in our study might be the specificity of the causality assessment and the assessment of drug-reaction relatedness in the database. Indeed, there is major effort in improving the quality 
Table 3 RORs related to antidepressant drugs and the PT "Upper gastrointestinal haemorrhage" with and without consideration of the competition bias with antithrombotic comedication

\begin{tabular}{|c|c|c|}
\hline \multirow[t]{2}{*}{ Substance } & \multicolumn{2}{|l|}{ ROR (95\% CI) } \\
\hline & $\begin{array}{l}\text { ROR without consideration of the com- } \\
\text { petition bias }\end{array}$ & $\begin{array}{l}\text { ROR with considera- } \\
\text { tion of the competition } \\
\text { bias }\end{array}$ \\
\hline Agomelatine & NA & NA \\
\hline Amitriptyline & NA & NA \\
\hline Bupropion & NA & NA \\
\hline Citalopram & $1.56(1.11-2.20)$ & $1.02(0.49-2.14)$ \\
\hline Clomipramine & NA & NA \\
\hline Doxepin & NA & NA \\
\hline Duloxetine & $0.39(0.23-0.66)$ & $0.59(0.28-1.23)$ \\
\hline Escitalopram & $1.52(1.03-2.25)$ & $0.56(0.18-1.73)$ \\
\hline Fluoxetine & $0.52(0.34-0.78)$ & $0.92(0.55-1.56)$ \\
\hline Fluvoxamine & NA & NA \\
\hline Hypericum perforatum & NA & NA \\
\hline Imipramine & NA & NA \\
\hline Lithium & NA & NA \\
\hline Maprotiline & NA & NA \\
\hline Milnacipran & NA & NA \\
\hline Mirtazapine & $0.28(0.11-0.75)$ & NA \\
\hline Moclobemide & NA & NA \\
\hline Nortriptyline & NA & NA \\
\hline Paroxetine & $0.83(0.59-1.16)$ & $0.86(0.49-1.52)$ \\
\hline Reboxetine & NA & NA \\
\hline Sertraline & $0.86(0.61-1.20)$ & $0.45(0.20-1.00)$ \\
\hline Tianeptine & NA & NA \\
\hline Tranylcypromine & NA & NA \\
\hline Trazodone & NA & NA \\
\hline Trimipramine & NA & NA \\
\hline Venlafaxine & $0.52(0.33-0.83)$ & $1.03(0.58-1.81)$ \\
\hline
\end{tabular}

$C I$ confidence interval, NA data not available (less than 3 records), $P T$ Preferred Term, ROR reporting odds ratio

of ICSRs [42]. However, there are several factors that need to be considered: one point is the information regarding causality. As the information in VigiBase ${ }^{\mathrm{TM}}$ is gathered by national pharmacovigilance centres worldwide, the causality assessment may differ according to the diverse practices of different countries. Furthermore, the assessment of causality may change over time in connection with increasing knowledge about a certain drug [43]. The reporting process is also prone to potential bias as the categorisation of the drug role/ probability of a causal relationship with the respective event as "suspected/interacting" or "concomitant" in the database is made by the primary reporter according to the International Council for Harmonisation of Technical Requirements for Pharmaceuticals for Human Use guideline [44]. This might be particularly relevant regarding SSRIs and non-steroidal anti-inflammatory drugs (NSAIDs) concerning gastrointestinal bleedings. Because there is a widely known relationship between NSAIDs and gastrointestinal bleeding
[45-47], one might, for example, report the NSAID taken by the patient as the "suspected/interacting" drug and the SSRI that is taken in parallel as "concomitant" or, in the worst case, might not even report the SSRI at all. To summarise, the use of the category "suspected/interacting", as used in our study (see Materials and Methods) may lead to the exclusion of several ADR reports of haemorrhages associated with ADs, particularly in cases where the reporting person is not aware of a possible causal involvement of the AD medication regarding the respective bleeding event; these cases may have been categorised as "concomitant" and need further investigation.

Unfortunately, in this work, we were not able consider the impact of NSAIDs regarding gastrointestinal bleeding events, which might also play a major role. In a systematic review and meta-analysis, Anglin et al. found a modest increase in the risk of upper gastrointestinal bleeding when SSRIs are combined with NSAIDs [25]. In our analysis, the 
primary goal was to investigate the effect of antithrombotics as competitors for signal detection in regard to an increased bleeding risk, as bleeding is a type A reaction for antithrombotics and thus might be relevant for the competition bias. The influence of NSAIDs on signal detection might require further investigation. However, one of the most commonly used NSAIDs, acetylsalicylic acid, was considered in our study because it is also an antithrombotic and listed in the ATC B01A. Another possible limitation in addition to the effects of NSAIDs is the potential influence of age, sex and the prior history of gastrointestinal bleeding, which might additionally affect the AD-associated bleeding risk [48, 49]. Still, we assume this should not have a major impact on the aim of this study because the primary outcome was the impact of antithrombotics on signal detection and the impact by age and sex might affect all cases of gastrointestinal bleeding, regardless of the intake of ADs.

\section{Conclusions}

Although the competition bias is generally a relevant factor in disproportionality analysis, we could not verify the hypothesis of a major impact of the competition bias (by considering the impact of reports related to antithrombotics) on the detection of the bleeding risk related to ADs. Therefore, antithrombotics seem unlikely to have a major impact on the detection of the bleeding risk of ADs in disproportionality analysis. The competition bias should be considered with caution, if the competing substance may have an impact in terms of a drug-drug interaction. Furthermore, the categorisation of the probability of a causal relationship between the drug of interest and the respective event plays a major role in disproportionality analysis and requires further evaluation.

Supplementary Information The online version contains supplementary material available at https://doi.org/10.1007/s40801-021-00260-9.

\section{Declarations}

Funding No funding was received for the conduct of this study or the preparation of this article.

Conflicts of interest/Competing interests René Zeiss, Christoph Hiemke, Carlos Schönfeldt-Lecuona, Bernhard J. Connemann and Maximilian Gahr have no conflicts of interest that are directly relevant to the content of this article. The research has not been presented or published elsewhere.

Ethics approval Not applicable, data from the UMC were received anonymised.

Consent to participate Not applicable.

Consent for publication Not applicable.
Availability of data and material The data used for the manuscript were extracted from VigiBase ${ }^{\mathrm{TM}}$, the World Health Organization global database of ICSRs.

Code availability Not applicable.

Authors' contributions All authors contributed to the study conception and design. Material preparation, data collection and analysis were performed by RZ and MG. The first draft of the manuscript was written by RZ and MG and all authors commented on previous versions of the manuscript. All authors read and approved the final manuscript.

Open Access This article is licensed under a Creative Commons Attribution-NonCommercial 4.0 International License, which permits any non-commercial use, sharing, adaptation, distribution and reproduction in any medium or format, as long as you give appropriate credit to the original author(s) and the source, provide a link to the Creative Commons licence, and indicate if changes were made. The images or other third party material in this article are included in the article's Creative Commons licence, unless indicated otherwise in a credit line to the material. If material is not included in the article's Creative Commons licence and your intended use is not permitted by statutory regulation or exceeds the permitted use, you will need to obtain permission directly from the copyright holder. To view a copy of this licence, visit http://creativecommons.org/licenses/by-nc/4.0/.

\section{References}

1. WHO. The importance of pharmacovigilance: safety monitoring of medicinal products. Geneva: WHO; 2002.

2. Montastruc JL, Sommet A, Bagheri H, et al. Benefits and strengths of the disproportionality analysis for identification of adverse drug reactions in a pharmacovigilance database. Br J Clin Pharmacol. 2011;72:905-8.

3. Meyboom RHB, Egberts ACG, Edwards IR, et al. Principles of signal detection in pharmacovigilance. Drug Saf. 1997;16:355-65.

4. Uppsala Monitoring Centre. UMC: what is a signal? 2017. https:// www.who-umc.org/research-scientific-development/signal-detec tion/what-is-a-signal/. Accessed 19 Sep 2017.

5. Bate A, Evans SJ. Quantitative signal detection using spontaneous ADR reporting. Pharmacoepidemiol Drug Saf. 2009;18:427-36.

6. Rawlins MD. Spontaneous reporting of adverse drug reactions. II: uses. Br J Clin Pharmacol. 1988;26:7-11.

7. Nguyen TTH, Pariente A, Montastruc JL, et al. An original pharmacoepidemiological-pharmacodynamic method: application to antipsychotic-induced movement disorders. Br J Clin Pharmacol. 2017;83:612-22.

8. Raschi E, Moretti U, Salvo F, et al. Evolving roles of spontaneous reporting systems to assess and monitor drug safety. Pharmacovigilance. 2019. https://doi.org/10.5772/intechopen. 79986.

9. Faillie JL. Case-non-case studies: principle, methods, bias and interpretation. Therapie. 2019;74:225-32.

10. Hazell L, Shakir SA. Under-reporting of adverse drug reactions: a systematic review. Drug Saf. 2006;29:385-96.

11. Pariente A, Gregoire F, Fourrier-Reglat A, et al. Impact of safety alerts on measures of disproportionality in spontaneous reporting databases: the notoriety bias. Drug Saf. 2007;30:891-8.

12. Pariente A, Didailler M, Avillach P, et al. A potential competition bias in the detection of safety signals from spontaneous reporting databases. Pharmacoepidemiol Drug Saf. 2010;19:1166-71. 
13. Pariente A, Avillach P, Salvo F, et al. Effect of competition bias in safety signal generation. Drug Saf. 2012;35:855-64.

14. Salvo F, Leborgne F, Thiessard F, et al. A potential eventcompetition bias in safety signal detection: results from a spontaneous reporting research database in France. Drug Saf. 2013;36:565-72.

15. National Institute for Health and Care Excellence. Depression in adults: treatment and management, London. 2018. https:// www.nice.org.uk/guidance/gid-cgwave0725/documents/fullguideline-updated. Accessed 30 May 2021.

16. Laporte $S$, Chapelle $C$, Caillet $P$, et al. Bleeding risk under selective serotonin reuptake inhibitor (SSRI) antidepressants: a meta-analysis of observational studies. Pharmacol Res. 2017;118:19-32.

17. Spina E, Barbieri MA, Cicala G, et al. Clinically relevant drug interactions between newer antidepressants and oral anticoagulants. Expert Opin Drug Metab Toxicol. 2020;16:31-44.

18. De Abajo FJ. Effects of selective serotonin reuptake inhibitors on platelet function: mechanisms, clinical outcomes and implications for use in elderly patients. Drugs Aging. 2011;28:345-67.

19. Andrade C, Sandarsh S, Chethan KB, et al. Serotonin reuptake inhibitor antidepressants and abnormal bleeding: a review for clinicians and a reconsideration of mechanisms. J Clin Psychiatry. 2010;71:1565-75.

20. Meijer WEE, Heerdink ER, Nolen WA, et al. Association of risk of abnormal bleeding with degree of serotonin reuptake inhibition by antidepressants. Arch Intern Med. 2004;164:2367-70.

21. Renoux C, Vahey S, Dell'Aniello S, et al. Association of selective serotonin reuptake inhibitors with the risk for spontaneous intracranial hemorrhage. JAMA Neurol. 2017;74:173-80.

22. Mahdanian AA, Looper KJ, Bacon SL, et al. Serotonergic antidepressants and increased bleeding risk in patients undergoing breast biopsy. Ther Adv Psychopharmacol. 2015;5:332-8.

23. Auerbach AD, Vittinghoff E, Maselli J, et al. Perioperative use of selective serotonin reuptake inhibitors and risks for adverse outcomes in surgery. JAMA Intern Med. 2013;173:1075-81.

24. Roose SP, Rutherford BR. Selective serotonin reuptake inhibitors and operative bleeding risk: a review of the literature. J Clin Psychopharmacol. 2016;36:704-9.

25. Anglin R, Yuan Y, Moayyedi P, et al. Risk of upper gastrointestinal bleeding with selective serotonin reuptake inhibitors with or without concurrent nonsteroidal anti-inflammatory use: a systematic review and meta-analysis. Am J Gastroenterol. 2014;109:811-9.

26. Loke YK, Trivedi AN, Singh S. Meta-analysis: gastrointestinal bleeding due to interaction between selective serotonin uptake inhibitors and non-steroidal anti-inflammatory drugs. Aliment Pharmacol Ther. 2008;27:31-40.

27. Hackam DG, Mrkobrada M. Selective serotonin reuptake inhibitors and brain hemorrhage: a meta-analysis. Neurology. 2012;79:1862-5.

28. Shin J-YY, Park M-JJ, Lee SH, et al. Risk of intracranial haemorrhage in antidepressant users with concurrent use of nonsteroidal anti-inflammatory drugs: nationwide propensity score matched study. BMJ. 2015;351:h3517.

29. Gahr M, Zeiss R, Lang D, et al. Risk of bleeding related to selective and non-selective serotonergic antidepressants: a case/ non-case approach using data from two pharmacovigilance databases. Pharmacopsychiatry. 2015;48:19-24.

30. Gahr M, Zeiss R, Lang D, et al. Association between haemorrhages and treatment with selective and non-selective serotonergic antidepressants: possible implications of quantitative signal detection. Psychiatry Res. 2015;229:257-63.

31. MedDRA. https://www.meddra.org/. Accessed 27 Mar 2019.

32. Tiaden JD, Wenzel E, Berthold HK, et al. Adverse reactions to anticoagulants and to antiplatelet drugs recorded by the german spontaneous reporting system. Semin Thromb Hemost. 2005;31:371-80.

33. Ferrajolo C, Capuano A, Verhamme KM, et al. Drug-induced hepatuc injury in children: a case/non-case study of suspected adverse drug reactions in VigiBase. Br J Clin Pharmacol. 2010;70:721-8.

34. Montastruc F, Khosrow-Khavar F, de Germay S, et al. Tamoxifen and the risk of parkinsonism: a case/non-case study. Eur J Clin Pharmacol. 2018;74:1181-4.

35. van Puijenbroek EP, Bate A, Leufkens HG, et al. A comparison of measures of disproporttionality for signal detection in spontaneous reporting systems for adverse drug reactions. Pharmacoepidemiol Drug Saf. 2002;11:3-10.

36. Rothman KJ, Lanes S, Sacks ST. The reporting odds ratio and its advantages over the proportional reporting ratio. Pharmacoepidemiol Drug Saf. 2004;13:519-23.

37. Schalekamp T, Klungel OH, Souverein PC, et al. Increased bleeding risk with concurrent use of selective serotonin reuptake inhibitors and coumarins. Arch Intern Med. 2008;168:180-5.

38. Schelleman H, Brensinger CM, Bilker WB, et al. Antidepressant-warfarin interaction and associated gastrointestinal bleeding risk in a case-control study. PLoS ONE. 2011;6:e21447.

39. Fernandez S, Lenoir C, Samer C, et al. Drug interactions with apixaban: a systematic review of the literature and an analysis of VigiBase, the World Health Organization database of spontaneous safety reports. Pharmacol Res Perspect. 2020;8:1-11.

40. Barbieri MA, Cutroneo PM, Baratelli C, et al. Adverse drug reactions with oral anticoagulants: data from sicilian spontaneous reporting system database. J Clin Pharm Ther. 2021. https:// doi.org/10.1111/jcpt.13391.

41. Monaco L, Biagi C, Conti V, et al. Safety profile of the direct oral anticoagulants: an analysis of the WHO database of adverse drug reactions. Br J Clin Pharmacol. 2017;83:1532-43.

42. Lindquist M. Data quality management in pharmacovigilance. Drug Saf. 2004;27:857-70.

43. Uppsala Monitoring Centre. Guideline for using VigiBase data in studies. Version. 2016;2:1-9.

44. International Council for Harmonisation of Technical Requirements for Pharmaceuticals for Human Use. Maintenance of the ICH guideline on clinical safety data management. E2B(R2), Geneva, Switzerland, 2001.

45. Allison MC, Howatson AG, Torrance CJ, et al. Gastrointestinal damage associated with the use of nonsteroidal antiinflammatory drugs. N Engl J Med. 1992;327:749-54.

46. Mellemkjaer L, Blot WJ, Sorensen HT, et al. Upper gastrointestinal bleeding among users of NSAIDs: a population-based cohort study in Denmark. Br J Clin Pharmacol. 2002;53:173-81.

47. Wolfe MM, Lichtenstein DR, Singh G. Gastrointestinal toxicity of nonsteroidal antiinflammatory drugs. N Engl J Med. 1999;340:1888-99.

48. Wang YP, Chen YT, Tsai CF, et al. Short-term use of serotonin reuptake inhibitors and risk of upper gastrointenstinal bleeding. Am J Psychiatry. 2014;171:54-61.

49. Wilkins T, Wheeler B, Carpenter M. Upper gastrointestinal bleeding in adults: evaluation and management. 2020. www.aafp.org/ afp. Accessed 13 Apr 2021. 